2015

\title{
Novel Phases Of Lithium-Aluminum Binaries From First-Principles Structural Search
}

Rafael Sarmiento-Pérez

Tiago F. T. Cerqueira

Irais Valencia-Jaime

Maximilian Amsler

Stefan Goedecker

See next page for additional authors

Follow this and additional works at: https://researchrepository.wvu.edu/faculty_publications

\section{Digital Commons Citation}

Sarmiento-Pérez, Rafael; Cerqueira, Tiago F. T.; Valencia-Jaime, Irais; Amsler, Maximilian; Goedecker, Stefan; Romero, Aldo H.; Botti, Silvana; and Marques, Miguel A. L., "Novel Phases Of Lithium-Aluminum Binaries From First-Principles Structural Search" (2015). Faculty Scholarship. 618.

https://researchrepository.wvu.edu/faculty_publications/618 
Authors

Rafael Sarmiento-Pérez, Tiago F. T. Cerqueira, Irais Valencia-Jaime, Maximilian Amsler, Stefan Goedecker, Aldo H. Romero, Silvana Botti, and Miguel A. L. Marques 


\section{A|P $\begin{aligned} & \text { The Journal of } \\ & \text { Chemical Physics }\end{aligned}$}

\section{Novel phases of lithium-aluminum binaries from first-principles structural search}

Rafael Sarmiento-Pérez, Tiago F. T. Cerqueira, Irais Valencia-Jaime, Maximilian Amsler, Stefan Goedecker, Aldo H. Romero, Silvana Botti, and Miguel A. L. Marques

Citation: The Journal of Chemical Physics 142, 024710 (2015); doi: 10.1063/1.4905141

View online: http://dx.doi.org/10.1063/1.4905141

View Table of Contents: http://scitation.aip.org/content/aip/journal/jcp/142/2?ver=pdfcov

Published by the AIP Publishing

\section{Articles you may be interested in}

High-temperature neutron diffraction and first-principles study of temperature-dependent crystal structures and atomic vibrations in Ti3AIC2, Ti2AIC, and Ti5Al2C3

J. Appl. Phys. 113, 183519 (2013); 10.1063/1.4803700

Effects of alloying elements and temperature on the elastic properties of dilute Ni-base superalloys from firstprinciples calculations

J. Appl. Phys. 112, 053515 (2012); 10.1063/1.4749406

Environment-dependent surface structures and stabilities of $\mathrm{SnO} 2$ from the first principles

J. Appl. Phys. 111, 063504 (2012); 10.1063/1.3694033

Alloying effects on the elastic parameters of ferromagnetic and paramagnetic Fe from first-principles theory J. Appl. Phys. 110, 073707 (2011); 10.1063/1.3644907

Interatomic potentials between distinct atoms from first-principles calculation and lattice-inversion method J. Appl. Phys. 82, 578 (1997); 10.1063/1.365618

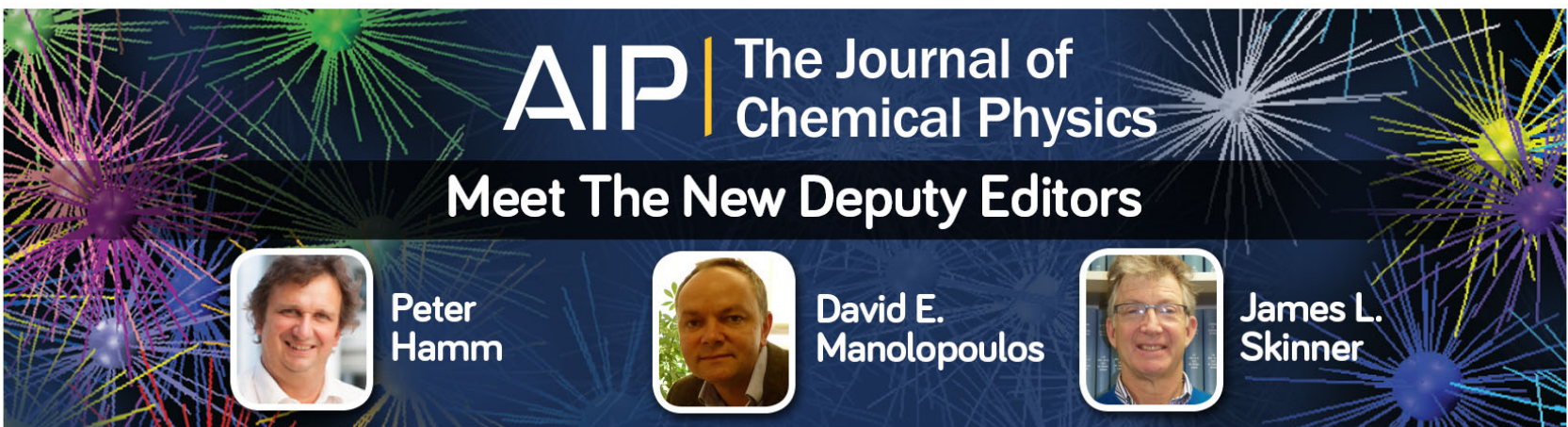




\title{
Novel phases of lithium-aluminum binaries from first-principles structural search
}

\author{
Rafael Sarmiento-Pérez, ${ }^{1}$ Tiago F. T. Cerqueira, ${ }^{1}$ Irais Valencia-Jaime, ${ }^{1,2}$ \\ Maximilian Amsler, ${ }^{3}$ Stefan Goedecker ${ }^{3}$ Aldo H. Romero, ${ }^{4}$ Silvana Botti, ${ }^{1}$ \\ and Miguel A. L. Marques $\left.{ }^{1, a}\right)$ \\ ${ }^{1}$ Institut Lumière Matière (UMR5306) and ETSF, Université Lyon 1-CNRS, Université de Lyon, \\ F-69622 Villeurbanne Cedex, France \\ ${ }^{2}$ Centro de Investigación y Estudios Avanzados del IPN, MX-76230 Querétaro, Mexico \\ ${ }^{3}$ Department of Physics, Universität Basel, Klingelbergstr. 82, 4056 Basel, Switzerland \\ ${ }^{4}$ Physics Department, West Virginia University, Morgantown, West Virginia 26506-6315, USA
}

(Received 1 August 2014; accepted 16 December 2014; published online 14 January 2015)

\begin{abstract}
Intermetallic $\mathrm{Li}-\mathrm{Al}$ compounds are on the one hand key materials for light-weight engineering, and on the other hand, they have been proposed for high-capacity electrodes for Li batteries. We determine from first-principles the phase diagram of Li-Al binary crystals using the minima hopping structural prediction method. Beside reproducing the experimentally reported phases ( $\mathrm{LiAl}, \mathrm{Li}_{3} \mathrm{Al}_{2}, \mathrm{Li}_{9} \mathrm{Al}_{4}$, $\mathrm{LiAl}_{3}$, and $\mathrm{Li}_{2} \mathrm{Al}$ ), we unveil a structural variety larger than expected by discovering six unreported binary phases likely to be thermodynamically stable. Finally, we discuss the behavior of the elastic constants and of the electric potential profile of all $\mathrm{Li}-\mathrm{Al}$ stable compounds as a function of their stoichiometry. @ 2015 AIP Publishing LLC. [http://dx.doi.org/10.1063/1.4905141]
\end{abstract}

\section{INTRODUCTION}

Light weight and good mechanical properties, together with attractive manufacturing costs, set $\mathrm{Al}$ alloys with $\mathrm{Li}$ at the forefront of aerospace materials research. The search for improved alloys is still open and attracts global interest for widespread industrial applications. It is well known that in the Al-rich range of stoichiometries ( $\mathrm{Li}$ concentrations between $5 \%$ and $25 \%$ ), $\mathrm{Li}-\mathrm{Al}$ alloys offer higher stiffness and superior strength-to-weight ratios $^{1-3}$ than pure Al. In fact, each additional weight percentage of Li reduces the density by about $3 \%$ while increasing the Young modulus by about $6 \%{ }^{2}$ This effect is explained by the hardening which occurs through the precipitation of nanoscale particles of the binary in the $\mathrm{Al}$ matrix. ${ }^{4}$ The strengthening nano-precipitates, known as the $\delta^{\prime}$ phase, are thought to be made of metastable $\mathrm{LiAl}_{3}$ with a cubic $(P m \overline{3} m)$ structure, which remains crystallographically coherent with the parent $\mathrm{Al}$ face-centered cubic (fcc) matrix with small lattice mismatch. Although some of the strengthening qualities originate from the precipitates acting as pinning centers for defects, it is proved by ab initio calculations that the stoichiometric $\mathrm{LiAl}_{3}$ crystal has a Young modulus significantly larger than $\mathrm{Al}^{5,6}$

Li-Al alloys have been investigated extensively, both experimentally and theoretically, and diverse applications have been proposed for this system. In literature, one can find information on five binary phases. While the Al-rich side of the phase diagram contains compounds with attractive elastic properties, the Li-rich side has been explored for high-capacity electrodes for Li batteries. ${ }^{7}$ The experimental data show that starting from a fcc metal (space group $F m \overline{3} m$ ) of pure $\mathrm{Al}$,

\footnotetext{
a)Electronic mail: marques@tddft.org
}

addition of Li produces fcc $\mathrm{Li}-\mathrm{Al}$ solid solutions until reaching the stoichiometry of the metastable simple cubic $\mathrm{LiAl}_{3}$ and then two stable binaries, the cubic $(F d \overline{3} m) \mathrm{LiAl}$ and, already in the $\mathrm{Li}$ rich range, the rhombohedral $(R \overline{3} m) \mathrm{Li}_{3} \mathrm{Al}_{2}$. The structure of $\mathrm{Li}_{3} \mathrm{Al}_{2}$ is indeed compatible with the rhombohedral $(R \overline{3} \mathrm{~m})$ zero temperature structure of Li. However, going from $\mathrm{Li}_{3} \mathrm{Al}_{2}$ to pure $\mathrm{Li}$, one passes through orthorhombic $(\mathrm{Cmcm})$ $\mathrm{Li}_{2} \mathrm{Al}$ and monoclinic $(C 2 / m) \mathrm{Li}_{9} \mathrm{Al}_{4} .{ }^{8}{ }^{89}$ The fact that at room temperature the crystal structure of pure $\mathrm{Li}$ becomes bodycenter cubic (bcc, $\operatorname{Im} \overline{3} m$ ) and that at $T=0 \mathrm{~K}$ the above mentioned rhombohedral, the bcc and a fcc phase are separated by less than $3 \mathrm{meV}$ per atom according to first-principles calculations is a clear indication of the complexity of bonding schemes that is brought by $\mathrm{Li}^{6}$

Theoretical investigations have mainly focused on the properties of the experimental phases and little has been done in order to identify new ones. Podloucky et al. ${ }^{5,10}$ studied Al-rich alloys using first-principles statistical-mechanics and electronic-structure approaches within the local density approximation $^{11}$ (LDA) to the exchange-correlation potential. They determined thermodynamical magnitudes considering a statistical ensemble of possible fcc supercells containing up to 8 atoms. Alam et al. ${ }^{12}$ also tried fcc and bcc-based compounds without finding any new stable structure. Also, using the Korringa-Kohn-Rostoker method within the coherentpotential approximation (KKR-CPA), they predicted a higher stability of off-stoichiometry Al-rich phases with respect to the ordered ones when the Li content is between $8 \%$ and $20 \%$.

The zero-temperature and zero-pressure binary phase diagram represents the thermodynamic equilibrium of phases of a two component system and it reveals useful insights into fundamental thermodynamical properties and into the variety of atomic coordination and bonding. Despite the large 
amount of studies in literature, the knowledge on the whole phase diagram of $\mathrm{Li}-\mathrm{Al}$ appears to be still incomplete. The experimental determination of a phase diagram is an extremely time-consuming process, which requires careful synthesis and characterization of all possible phases. On the other hand, first-principles calculations of phase diagrams of binary alloys remained for long an unreachable goal. Nowadays, accurate $a b$ initio computational tools based on density functional theory (DFT) can significantly accelerate the construction of phase diagrams. Furthermore, it is straightforward to include the effects of pressure and temperature.

\section{THEORETICAL METHOD}

Determining a binary phase-diagram requires to find the lowest-energy structures for a large range of different possible binary compositions. The best way to search for new crystal structures is to perform a systematic survey of the enthalpy surface using some sophisticated crystal-structure prediction method. In this context, we use the minima hopping method $(\mathrm{MHM})^{13,14}$ which employs a very efficient algorithm to identify the global minimum on the enthalpy surface while gradually exploring low-lying structures. This method has proved to be able to reproduce experimental results and to find novel structures starting only from the atomic composition of the system. ${ }^{15-21}$ The MHM algorithm, here coupled to DFT, explores the ground-state energy surface by performing short molecular dynamics escape steps followed by local geometry relaxations, taking into account both atomic and cell variables. The initial velocities for the dynamics are aligned preferably along soft-mode directions in order to favor the escape through low barriers to low-enthalpy structures. Revisiting already known structures is avoided by a feedback mechanism.

\section{RESULTS AND DISCUSSION}

In this work, we apply the MHM to study the phase diagram of $\mathrm{Li}_{x} \mathrm{Al}_{(x-1)}$, exploring 33 different stoichiometries from pure $\mathrm{Al}$ to pure Li. For a given stoichiometry, the initial geometries for the MHM runs were obtained randomly, ensuring only that the minimal distance between the atoms was at least equal to the sum of the covalent radii. We used cells containing up to 13 atoms, which, depending on the stoichiometry, corresponds to 1-5 formula units. Forces and energies were obtained in the framework of DFT using the all-electron projector augmented wave method as implemented in VASP. ${ }^{22}$ We used the Perdew-Burke-Ernzerhof (PBE) approximation ${ }^{23}$ to the exchange-correlation functional, together with a planewave cutoff of $520 \mathrm{eV}$ and number of k-points sufficient to guarantee a numerical convergence of total energies to less than $2 \mathrm{meV} /$ atom.

With the lowest energies structures obtained from the MHM calculations, we constructed the theoretical convex hull which is presented in Fig. 1 with a black line. To showcase the performance of our method in predicting crystal structures, we include in Fig. 1 also the convex hull constructed with the energy of the previous known Li-Al phases (red line). Most of these were available through the AFLOWLIB ${ }^{24}$ and

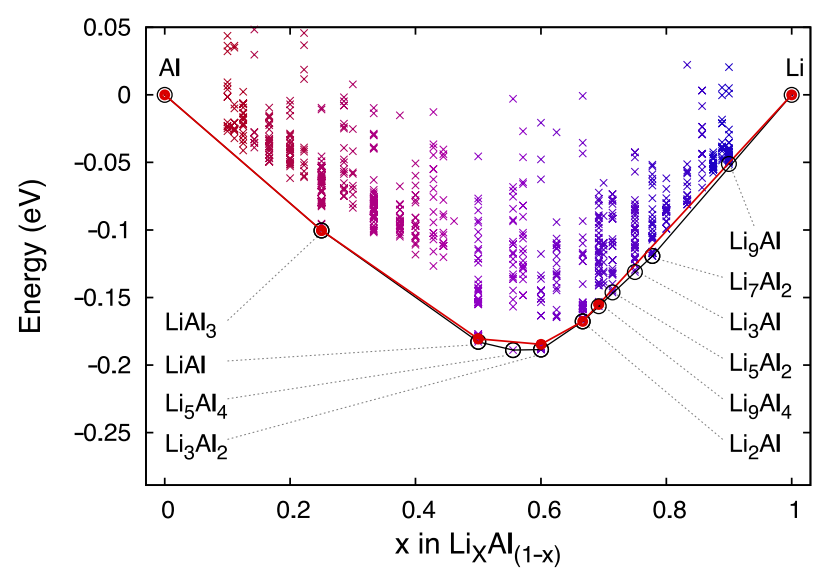

FIG. 1. Convex hull of the Li-Al binary system. Black line: convex hull constructed from the ab initio MHM calculations. Red line: convex hull constructed from the experimentally known structures. Crosses indicate different metastable phases identified in the MHM runs. The thermodynamically stable compositions are indicated by circles: red filled: experimental structures; black: novel structures.

OQMD ${ }^{25}$ databases, while the structure of $\mathrm{Li}_{2} \mathrm{Al}$ was taken from Refs. 8 and 9. The energy of the thermodynamically stable compositions, which form the convex hull, are marked with a circle. Red filled circles refer to experimental structures and the crosses indicate the energies of the low-lying local minima explored by the MHM algorithm during our simulations. To select the stable compositions, we consider the structures with energies close (within $8 \mathrm{meV} /$ atom) to the convex hull as belonging to it. In this way, we take into account effects like the inherent errors in the estimation of the formation energy, temperature, pressure, and defects, which may slightly modify the position of the structures with respect to the stability lines.

In order to evaluate the effect of the choice for the exchange-correlation functional on the convex hull, we repeated the reoptimization step using different approximations, namely, the LDA, ${ }^{11} \mathrm{PBEsol}^{26}$ (a revised PBE functional that improves equilibrium properties of densely packed solids and their surfaces), and $\mathrm{HSE}^{27,28}$ at the PBEsol geometries. The results are shown in Fig. 2, where we can see that the shape of the

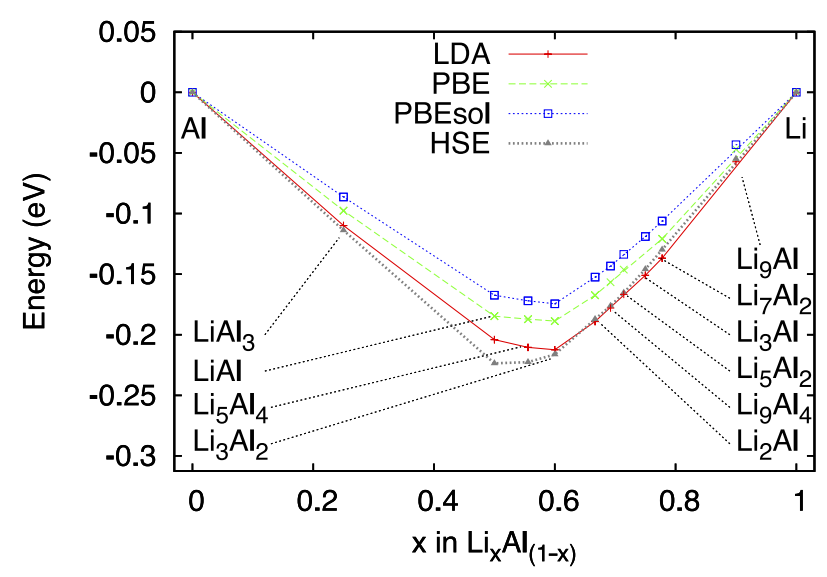

FIG. 2. Convex hull of the Li-Al binary system using different exchangecorrelation functionals. Red line with plus: LDA calculations. Green line with crosses: PBE calculations. Blue line with squares: PBEsol calculations. Dark grey line with triangles: HSE calculations at the PBEsol geometries. The thermodynamically stable compositions are indicated by the symbols on the convex hull lines. 
TABLE I. Lowest-energy phases obtained from the MHM calculations. Distances and angles are given in ångström and degree, respectively.

\begin{tabular}{|c|c|c|c|c|c|c|c|}
\hline Phase & Space group & $a$ & $b$ & $c$ & $\alpha$ & $\beta$ & $\gamma$ \\
\hline $\mathrm{Al}$ & $F m \overline{3} m$ & 4.041 & 4.041 & 4.041 & 90.00 & 90.00 & 90.00 \\
\hline $\mathrm{LiAl}_{3}$ & $P m \overline{3} m$ & 4.025 & 4.025 & 4.025 & 90.00 & 90.00 & 90.00 \\
\hline $\mathrm{LiAl}$ & $F d \overline{3} m$ & 6.348 & 6.348 & 6.348 & 90.00 & 90.00 & 90.00 \\
\hline $\mathrm{Li}_{5} \mathrm{Al}_{4}$ & $P \overline{3} m 1$ & 4.446 & 4.446 & 8.391 & 90.00 & 90.00 & 120.00 \\
\hline $\mathrm{Li}_{3} \mathrm{Al}_{2}$ & $R \overline{3} m$ & 4.446 & 4.446 & 14.073 & 90.00 & 90.00 & 120.00 \\
\hline $\mathrm{Li}_{2} \mathrm{Al}$ & $\mathrm{Cmcm}$ & 4.592 & 9.596 & 4.448 & 90.00 & 90.00 & 90.00 \\
\hline $\mathrm{Li}_{2} \mathrm{Al}$ & $P 6 / \mathrm{mmm}$ & 4.541 & 4.541 & 2.667 & 90.00 & 90.00 & 120.00 \\
\hline $\mathrm{Li}_{9} \mathrm{Al}_{4}$ & $C 2 / m$ & 5.367 & 4.453 & 18.633 & 105.41 & 103.14 & 90.17 \\
\hline $\mathrm{Li}_{5} \mathrm{Al}_{2}$ & $C 2 / m$ & 5.401 & 4.465 & 9.698 & 90.00 & 95.27 & 90.00 \\
\hline $\mathrm{Li}_{3} \mathrm{Al}$ & $P 2_{1} / m$ & 5.420 & 4.460 & 5.636 & 90.00 & 100.03 & 90.00 \\
\hline $\mathrm{Li}_{7} \mathrm{Al}_{2}$ & $R \overline{3} m$ & 7.932 & 7.932 & 8.226 & 90.00 & 90.00 & 120.00 \\
\hline $\mathrm{Li}_{9} \mathrm{Al}$ & $P \overline{1}$ & 5.076 & 5.093 & 7.936 & 83.45 & 71.71 & 70.30 \\
\hline $\mathrm{Li}$ & $R \overline{3} m$ & 3.067 & 3.067 & 22.085 & 90.00 & 90.00 & 120.00 \\
\hline
\end{tabular}

convex hull is to a large extent independent of the choice for the exchange-correlation potential, and therefore the same compositions are thermodynamically stable for all the tested approximations.

The stable crystal structures are summarized in Table I and the atomic arrangements of the most significant phases are displayed in Figs. 3 and 4. We found eleven stable intermetallic structures, out of which six had not been reported before. The other five are the experimentally known structures mentioned above. For these, the lattice constants were determined in very good agreement with the experimental values. ${ }^{6,8,9,29,30}$ It is important to stress that the MHM algorithm was able to find all the experimentally known structures and to determine new

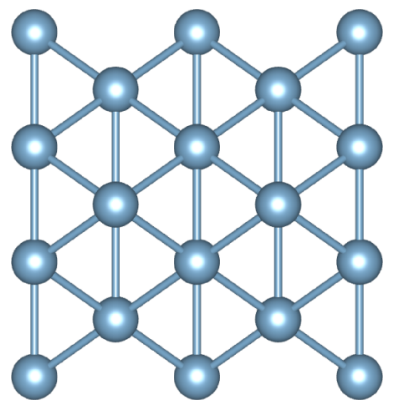

(a)

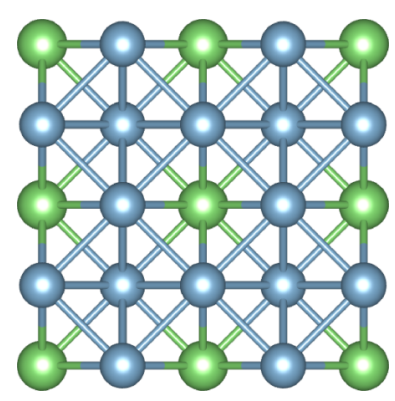

(c)

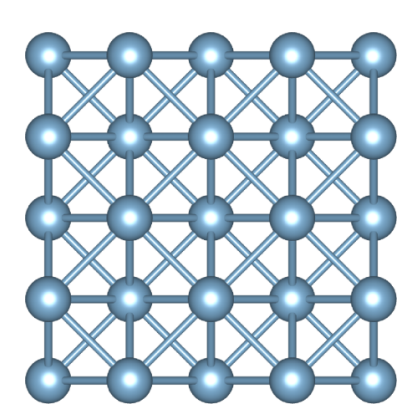

(b)

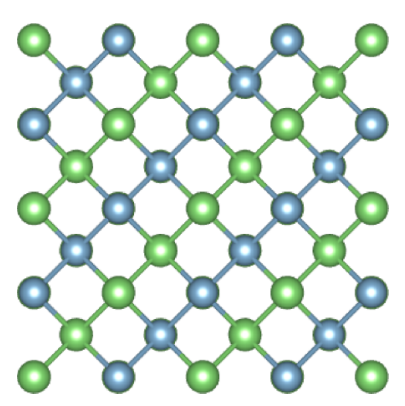

(d)
FIG. 3. Crystal structures of the Al-rich phases of Li-Al: (a) Al (110) surface (fcc, $F m \overline{3} m$ ); (b) Al (100) surface (fcc, $F m \overline{3} m$ ); (c) $\mathrm{LiAl}_{3}$ (fcc, $P m \overline{3} m$ ); (d) LiAl (fcc, $F d \overline{3} m$ ). Li atoms are green (lighter gray) while Al atoms are blue (darker gray). phases lower in energy than the ones available in the databases. This confirms its predictive power, which is especially relevant in this system that presents a large diversity of atomic arrangements.

From a careful analysis of Fig. 1, we can notice that almost straight lines connect $\mathrm{LiAl}$ with pure $\mathrm{Al}$, passing through $\mathrm{LiAl}_{3}$, that touches the convex hull. This is in agreement with the known metastability of $\mathrm{LiAl}_{3}$ : since it lies on a straight line, it will likely coexist with pure $\mathrm{Al}$ and $\mathrm{LiAl}$. The same occurs on the straight line connecting $\mathrm{Li}_{3} \mathrm{Al}_{2}$ and $\mathrm{Li}_{7} \mathrm{Al}_{2}$ : the phases lying on the convex hull between these two compounds can easily coexist in mixtures of the two extreme compositions. We can conclude that $\mathrm{LiAl}, \mathrm{Li}_{3} \mathrm{Al}_{2}$, and $\mathrm{Li}_{5} \mathrm{Al}_{4}$ are the most stables binary phases. Note that $\mathrm{Li}_{5} \mathrm{Al}_{4}$ is absent from known databases. Even if most of the $\mathrm{Li}-\mathrm{Al}$ compounds that we identify as thermodynamically stable can easily decompose, they will likely exist as microscopic inclusions in a matrix of another stoichiometry, contributing therefore to the physical properties of the alloy. It is therefore relevant to study the structural, elastic, and electronic properties of the $\mathrm{Li}-\mathrm{Al}$ lowest-energy binaries all over the composition range.

Figure 3 shows the atomic arrangements of the compounds that we found stable in the $\mathrm{Al}$ rich range of the phase diagram. The two stable crystal structures of $\mathrm{LiAl}_{3}$ and $\mathrm{LiAl}$ can be easily related to the original fcc Bravais lattice of $\mathrm{Al}$. $\mathrm{LiAl}_{3}$ is a simple cubic crystal with 4 atoms in the unit cell, where $\mathrm{Li}$ occupy the vertices of the cubes and $\mathrm{Al}$ the center of the cube faces, recreating the original fcc lattice but with two different atoms on the lattice sites. The $\mathrm{Al}-\mathrm{Al}$ and $\mathrm{Al}-\mathrm{Li}$ distances are $2.85 \AA$, basically unmodified with respect to fcc Al. We can therefore see that this crystal structure is perfectly compatible with the starting fcc lattice of $\mathrm{Al}$. LiAl is made of two interpenetrating fcc lattices, one made of $\mathrm{Li}$ and the other one of $\mathrm{Al}$, displaced by (0.5 0.5 0.5) $a$, where $a$ is the length of the conventional cube. In this structure, each atom has an equal number of $\mathrm{Al}$ and $\mathrm{Li}$ neighbors. $\mathrm{Li}-\mathrm{Li}$ distances and $\mathrm{Al}-\mathrm{Al}$ distances measure $2.75 \AA$, which are significantly smaller than the bond length in pure $\mathrm{Al}(2.86 \AA)$ and pure $\mathrm{Li}(3.05 \AA)$.

The $\mathrm{Li}$ rich side of $\mathrm{Li}-\mathrm{Al}$ alloys is more interesting concerning the complexity of the ground-state structures, with intermetallic compounds that differ significantly from their 


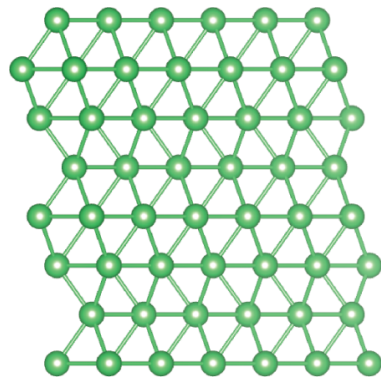

(a)

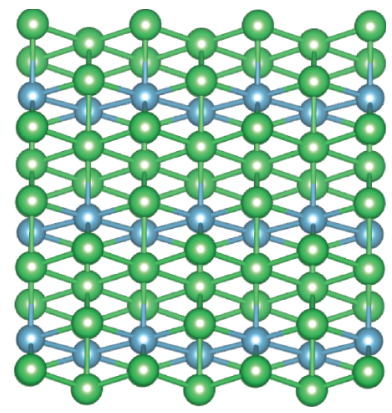

(c)

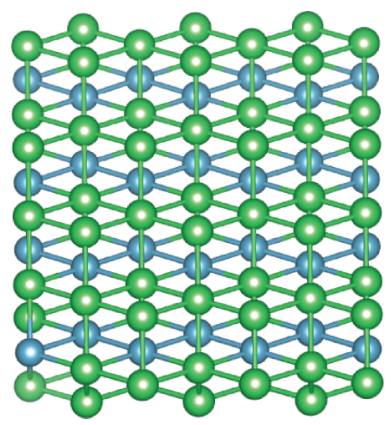

(e)

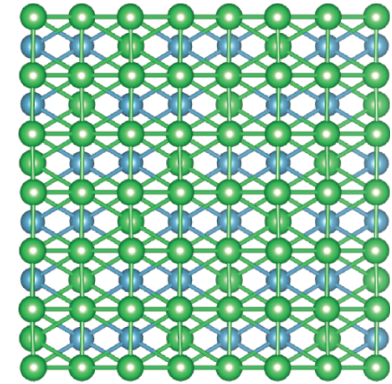

(b)

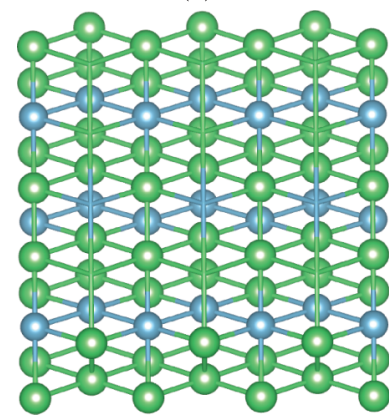

(d)

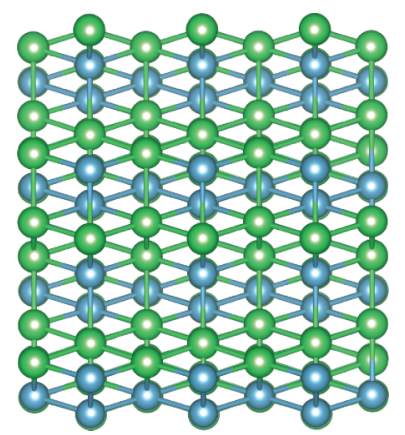

(f)
FIG. 4. Crystal structures of the Li-rich phases of Li-Al: (a) Li (rhombohedral, $R \overline{3} m$ ); (b) $\mathrm{Li}_{7} \mathrm{Al}_{2}$ (rhombohedral, $R \overline{3} m$ ); (c) $\mathrm{Li}_{5} \mathrm{Al}_{2}$ (monoclinic, $C 2 / m$ ); (d) $\mathrm{Li}_{2} \mathrm{Al}$ (monoclinic, $P 2_{1} / m$ ); (e) $\mathrm{Li}_{3} \mathrm{Al}_{2}$ (rhombohedral, $R \overline{3} m$ ); (f) $\mathrm{Li}_{5} \mathrm{Al}_{4}$ (trigonal, $\overline{3} \mathrm{~m} 1$ ). $\mathrm{Li}$ atoms are green while $\mathrm{Al}$ atoms are blue.

parent pure crystals. On this side of the phase diagram, we found several stable or almost stable compounds for a Li content greater than 50 at. \% that were not reported in literature. In Fig. 4, we show some phases belonging to the Li side of the phase diagram. We remind that Li has a rhombohedral $R \overline{3} m$ ground state structure at zero temperature characterized by a $\mathrm{Li}-\mathrm{Li}$ bond length of $3.05 \AA$, but fcc and bcc structures are only few meV per atom higher in energy. This gives a hint that the structural variety can be much larger for Li-rich compounds. $\mathrm{Li}_{9} \mathrm{Al}$ has a distorted bcc structure, with one out of 10 cube centers occupied by Li instead of Al. The bond length between $\mathrm{Li}$ atoms is basically unchanged with respect to pure $\mathrm{Li}$. $\mathrm{Li}_{7} \mathrm{Al}_{2}$ and $\mathrm{Li}_{3} \mathrm{Al}_{2}$ are rhombohedral, $\mathrm{Li}_{5} \mathrm{Al}_{4}$ is trigonal, and $\mathrm{Li}_{3} \mathrm{Al}$ has several orthorhombic and monoclinic phases within few meV from the hull.

Indeed, while we can observe on the $\mathrm{Al}$ rich side of the phase diagram of Fig. 1 that the lowest energy minima are clearly separated from the other geometrically distinct minima by sizable energy gaps, Li rich compounds show a quasicontinuous distribution of low-lying minima. This suggests that $\mathrm{Al}$ rich ordered crystals with small unit cells should be easier to obtain experimentally than Li rich crystals. In the latter case, one can rather expect at finite temperature the coexistence of different low-energy structures, possibly in a disordered phase.

Concerning the stoichiometry $\mathrm{Li}_{2} \mathrm{Al}$, which is experimentally reported as an orthorhombic crystal ${ }^{9}$ with space group Cmcm, we could find a hexagonal $P 6 / \mathrm{mmm}$ and a trigonal $P \overline{3} m 1$ structure which are only 2 and $5 \mathrm{meV} /$ atom higher in energy, respectively. In the hexagonal phase, Li atoms are arranged in aligned honeycomb lattice layers, forming hexagonal cells with one $\mathrm{Al}$ in the center. In the orthorhombic structure, $\mathrm{Al}$ atoms are instead arranged in zigzag chains, while the trigonal phase contains zigzag planes of Al. Also in this case, all three structures (and relative mixed configurations) must be considered as possible stable phases due to the very small energy differences.

The structures with about 50 at. $\%$ of $\mathrm{Li}$ are peculiar as the bond lengths are very different with respect to both parent $\mathrm{Li}$ and $\mathrm{Al}$ compounds: $\mathrm{Li}-\mathrm{Li}, \mathrm{Al}-\mathrm{Al}$, and $\mathrm{Li}-\mathrm{Al}$ bonds are contracted compared to the neighboring compositions. In general, the $\mathrm{Li}-\mathrm{Li}$ bond is reduced in all compounds on the $\mathrm{Li}$ rich side except for $\mathrm{Li}_{9} \mathrm{Al}$. We can also observe that $\mathrm{Al}$ atoms tends to have other $\mathrm{Al}$ as nearest neighbors even for Li-rich stoichiometries. $^{6}$

To further study the stability of the new phases, we also calculated phonon bands. Frozen phonons calculations were performed with the software PHONOPY from force constants calculated using vasP for $2 \times 2 \times 2$ supercells with finite displacements. All forces were obtained within the LDA. Figure 5 shows phonon band structures for a selection of stable phases. Similar phonon band structures were obtained for the other stable stoichiometries and they are included as supplementary material. ${ }^{31}$ No unstable modes were identified. Although this does not guarantee that the identified structures constitute the ground state at each composition, it validates them as dynamically stable structures.

In view of the importance of the mechanical properties of Li-Al alloys, we calculated the dependence of the atomic density and of the elastic constants as a function of Li content. As we want to simulate polycrystalline samples, that can be macroscopically described as isotropic elastic materials, we used appropriate averages of the elastic constants. The behaviour of polycrystalline linear elastic materials can be described with a set of two independent parameters. Here, we select the bulk modulus $B$ and the shear modulus $G$ and we estimate them using the Voigt-Reuss-Hill (VRH) averaging scheme. ${ }^{32}$ Voigt and Reuss averages give, respectively, upper and lower boundaries for the elastic constants. ${ }^{32}$ If the values obtained in the two approaches are close, their arithmetic media is a reliable estimate of the searched quantity. Once that $B$ and $G$ are determined, it is easy to calculate the Young modulus $E$ and Poisson's ratio $v$, using $G=E /(2+2 v)$ and $B=E /(3$ $-6 v)$. The elastic constants were calculated from the stress and strain relations by performing six distortions of the lattice, as implemented in vASP. In order to assure the convergence of all quantities by less than $1 \mathrm{GPa}$, we had to increase the kinetic energy cutoffs to $1000 \mathrm{eV}$ and the k-point meshes to $15 \times 15 \times 15$. The results are summarized in Table II, where we report the calculated values for the bulk modulus $B$, the 

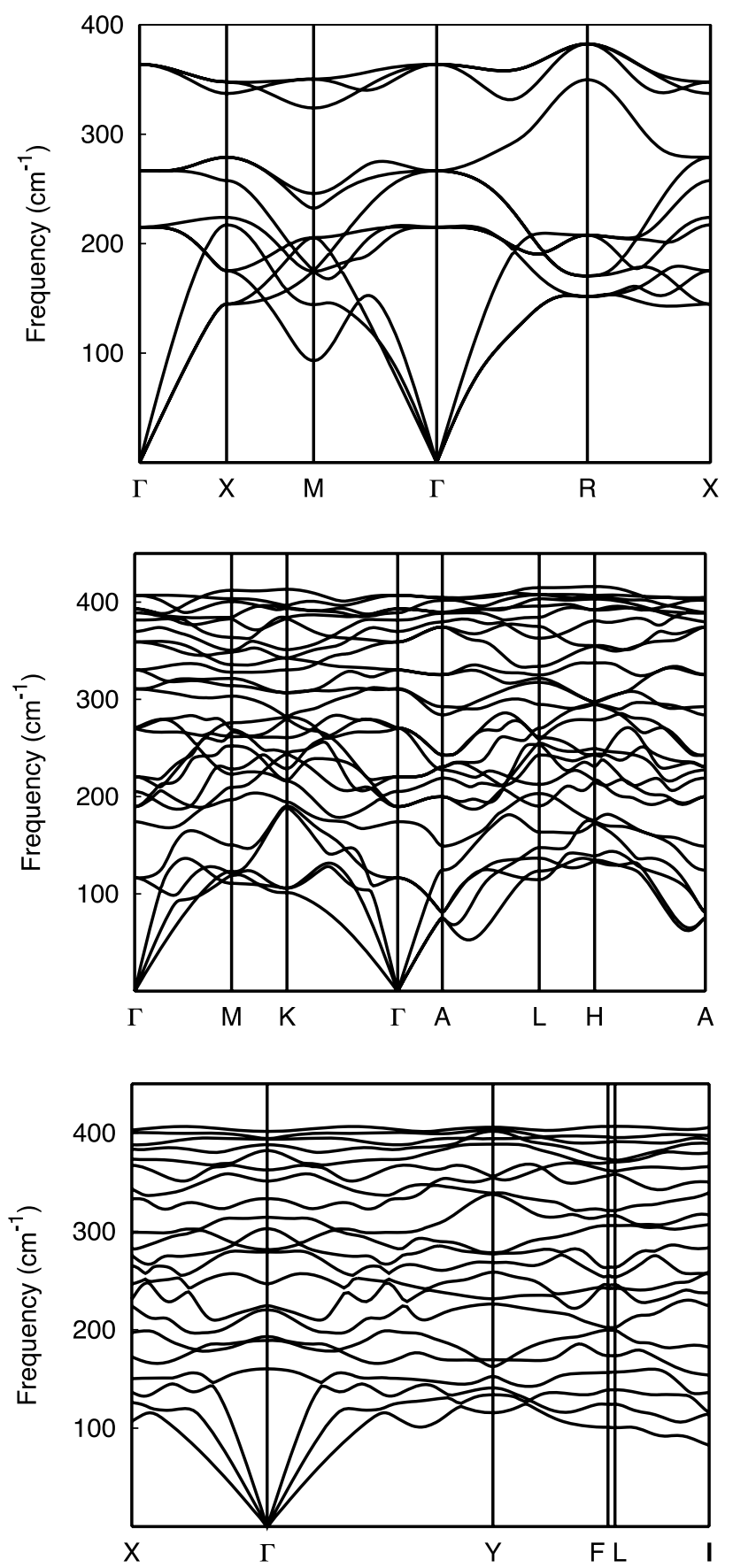

FIG. 5. Phonon band structures of the phases $\mathrm{Li}_{1} \mathrm{Al}_{3}$ (top), $\mathrm{Li}_{5} \mathrm{Al}_{4}$ (middle), and $\mathrm{Li}_{5} \mathrm{Al}_{2}$ (bottom).

shear modulus $G$, the Young modulus $E$, and the Poisson's ratio $v$ of all stable phases. We remark that these calculations are performed using a PBE exchange-correlation functional. We also tested the LDA and PBEsol functionals, obtaining variations of the values of the elastic constants smaller than $10 \%$.

In Fig. 6, we show the dependence of the density and the elastic moduli on the $\mathrm{Li}$ concentration. The calculated moduli are in reasonably good agreement with available experiments. ${ }^{33,34}$ The new structures meet the properties that were already reported: when increasing the amount of $\mathrm{Li}$ the bulk modulus ${ }^{35,36}$ decreases almost monotonically with
TABLE II. Calculated bulk $(B)$, shear $(G)$, Young $(E)$ moduli in GPa, Poisson's ratio $(v)$ and $B / G$ for the different phases. Experimental values are given for pure $\mathrm{Li}$ and $\mathrm{Al}$.

\begin{tabular}{|c|c|c|c|c|c|}
\hline Phase & $B$ & $G$ & $E$ & $v$ & $B / G$ \\
\hline $\mathrm{Al}$ & 78.1 & 30.5 & 80.9 & 0.33 & 2.6 \\
\hline Expt. (Ref. 33) & 75.86 & 28.34 & 75.60 & 0.33 & 2.7 \\
\hline $\mathrm{LiAl}_{3}$ & 63.4 & 39.8 & 98.7 & 0.24 & 1.6 \\
\hline LiAl & 47.6 & 30.9 & 75.7 & 0.24 & 1.5 \\
\hline $\mathrm{Li}_{5} \mathrm{Al}_{4}$ & 41.8 & 33.6 & 79.0 & 0.19 & 1.2 \\
\hline $\mathrm{Li}_{3} \mathrm{Al}_{2}$ & 38.9 & 32.8 & 76.4 & 0.17 & 1.2 \\
\hline $\mathrm{Li}_{2} \mathrm{Al}$ & 34.9 & 33.1 & 75.4 & 0.14 & 1.1 \\
\hline $\mathrm{Li}_{2} \mathrm{Al}$ & 32.6 & 27.1 & 63.3 & 0.18 & 1.2 \\
\hline $\mathrm{Li}_{9} \mathrm{Al}_{4}$ & 31.8 & 25.4 & 59.8 & 0.19 & 1.3 \\
\hline $\mathrm{Li}_{5} \mathrm{Al}_{2}$ & 29.0 & 22.6 & 53.4 & 0.19 & 1.3 \\
\hline $\mathrm{Li}_{3} \mathrm{Al}$ & 27.8 & 21.6 & 51.2 & 0.19 & 1.3 \\
\hline $\mathrm{Li}_{7} \mathrm{Al}_{2}$ & 27.6 & 21.7 & 51.5 & 0.19 & 1.3 \\
\hline $\mathrm{Li}_{9} \mathrm{Al}$ & 18.4 & 9.4 & 23.5 & 0.29 & 2.0 \\
\hline Li (bcc) & 12.9 & 5.5 & 14.0 & 0.32 & 2.3 \\
\hline Expt. (Ref. 34) & 12.13 & 8.78 & 21.22 & 0.21 & 1.4 \\
\hline
\end{tabular}

the volumetric mass density (not shown in Fig. 6). This is consistent with a much stronger resistance of $\mathrm{Al}$ to volume changes by applied pressure. However, the Young modulus increases on the Al-rich side of the phase diagram by small addition of $\mathrm{Li}^{1-3}{ }^{1-3}$ in apparent contrast with the fact that the Young modulus of $\mathrm{Li}$ is 5-6 times smaller than the one of Al. We further notice that the Young modulus of $\mathrm{LiAl}_{3}$ is considerably higher than that of pure $\mathrm{Al}$, and that the Young modulus is essentially constant up to concentrations of $60 \%$ of $\mathrm{Li}$, when it starts decreasing at a fast rate. A similar dependence on the composition is shown by the atomic density, which is also plotted for comparison in Fig. 6. The Young modulus is together with the shear modulus a good estimate of the stiffness of the material. This shows that, from a purely mechanical point of view, alloys containing substantially more than $5 \%-25 \%$ of Li could be used without deterioration of the stiffness.

Also in the context of Li batteries, the mechanical properties of the electrodes are important to prevent mechanical failures as a consequence of the stress developed due to large

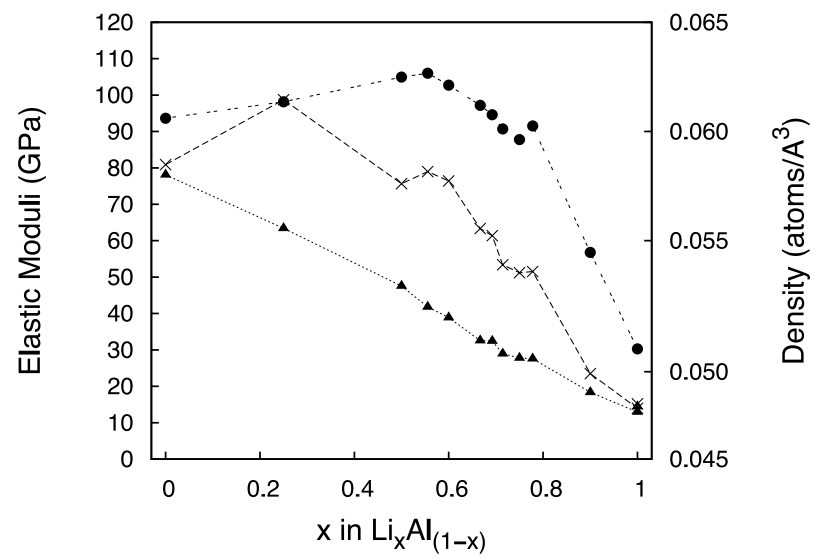

FIG. 6. Bulk modulus (filled triangles, in GPa), Young modulus (crosses, in $\mathrm{GPa}$ ), and atomic number density (filled circles, in atoms $/ \AA^{3}$ ) as a function of the $\mathrm{Li}$ concentration. 
volume expansion upon lithiation. The ratio between the bulk modulus and shear modulus gives an estimate of the ductile or brittle behavior of a material: $B / G$ ratios smaller than 1.75 indicate brittle alloys, while $B / G$ is larger than 1.75 in ductile alloys. Most of the considered structures are brittle. Beside the obvious exception of pure $\mathrm{Al}$, which is ductile, also $\mathrm{Li}_{9} \mathrm{Al}$ and $\mathrm{Li}$ have a $B / G$ ratio larger than 1.75 . Nevertheless, by comparison with the experimental value of $B / G$ for $\mathrm{Li}$ (1.4), we understand that this is an artifact due to the coincidental overestimation of $B$ and underestimation of $G$. These results point to the risk of mechanical failures in LiAl alloys when used for electrodes. ${ }^{37}$ The Poisson's ratio $v$ is defined as the ratio of transverse strain to the longitudinal strain and it gives information on the resistance of the material against shear, therefore giving indirect access to bonding forces. The value of the $v$ is usually in the interval $0-0.5$, and larger values of $v$ reflect better plasticity. Our results show an average plasticity with a better behavior while approaching pure $\mathrm{Al}$ and pure $\mathrm{Li}$.

The phase stability and the bonding characteristics of the alloys can be better understood by looking at the distribution of valence charge around each atom. A characteristic of Alrich compounds is that $\mathrm{Li}$ atoms redistribute some of their valence electrons to the $\mathrm{Al}$ bonds and the resultant strengthened $\mathrm{Al}$ bonds stabilize the compounds. We observed this redistribution of charge (except for $\mathrm{Li}_{9} \mathrm{Al}$ where the quantity of $\mathrm{Al}$ is too small) by calculating the Bader charges for all the studied compounds. This added stabilization can be easily seen by plotting the atomic number density of the different compositions, measured in atoms per unit volume. As we see in Fig. 6, there is an excellent correlation between the Young modulus and the atomic number density. (Note that this is in contrast with the usual volumetric mass density that decreases almost linearly as a function of increasing Li concentration.) In fact, up to concentrations of $60 \%$ of $\mathrm{Li}$, the increased bonding leads to denser and therefore stiffer structures. This effect is maximized for $\mathrm{LiAl}_{3}$.

\section{CONCLUSIONS}

In summary, we have discovered several unknown ordered phases of the Al-Li binary system, which are thermodynamically stable. Phonon band structures indicate that these new $\mathrm{Li}-\mathrm{Al}$ structures are also dynamically stable. Analysis of the elastic constants indicate that the stiffness of LiAl alloys with up to $60 \%$ of $\mathrm{Li}$ remains essentially equal to the one of $\mathrm{Al}$, with a marked maximum at $\mathrm{LiAl}_{3}$. This can be understood by the stabilization of these compounds (due to a transfer from $\mathrm{Li}$ atoms to $\mathrm{Al}$ bonds), which increases the atomic number density and therefore the stiffness. These results expand greatly our knowledge of the $\mathrm{Li}-\mathrm{Al}$ phase diagram and can have a profound influence in the understanding and design of new $\mathrm{Li}-\mathrm{Al}$ alloys for light-weight engineering.

\section{ACKNOWLEDGMENTS}

M.A.L.M. and S.B. acknowledge financial support from the French ANR Project No. ANR-12-BS04-0001-02. Computational resources were provided by GENCI (Project No. x2014096017). A. H. Romero and I. Valencia-Jaime acknowledge the support of the Marie Curie Actions from the European Union in the international incoming fellowships (Grant No. PIIFR-GA-2011-911070) and the Donors of the American Chemical Society Petroleum Research Fund for partial support of this research under Contract No. 54075-ND10.

${ }^{1}$ B. Noble, S. Harris, and K. Dinsdale, J. Mater. Sci. 17, 461 (1982).

${ }^{2}$ E. Lavernia and N. Grant, J. Mater. Sci. 22, 1521 (1987).

${ }^{3}$ E. Lavernia, T. Srivatsan, and F. Mohamed, J. Mater. Sci. 25, 1137 (1990).

${ }^{4}$ J. Laverock, S. B. Dugdale, M. A. Alam, M. V. Roussenova, J. R. Wensley, J. Kwiatkowska, and N. Shiotani, Phys. Rev. Lett. 105, 236401 (2010).

${ }^{5}$ X.-Q. Guo, R. Podloucky, J.-h. Xu, and A. J. Freeman, Phys. Rev. B 41, 12432 (1990).

${ }^{6}$ X.-Q. Guo, R. Podloucky, and A. J. Freeman, Phys. Rev. B 42, 10912 (1990).

${ }^{7}$ Y. Hamon, T. Brousse, F. Jousse, P. Topart, P. Buvat, and D. M. Schleich, J. Power Sources 97, 185 (2001).

${ }^{8}$ A. McAlister, Bull. Alloy Phase Diagrams 3, 177 (1982).

${ }^{9}$ K. Puhakainen, M. Bostrom, T. L. Groy, and U. Haussermann, J. Solid State Chem. 183, 2528 (2010).

${ }^{10}$ R. Podloucky, H. J. F. Jansen, X. Q. Guo, and A. J. Freeman, Phys. Rev. B 37, 5478 (1988).

${ }^{11}$ W. Kohn and L. J. Sham, Phys. Rev. 140, A1133 (1965).

${ }^{12}$ A. Alam and D. D. Johnson, Phys. Rev. B 85, 144202 (2012).

${ }^{13}$ S. Goedecker, J. Chem. Phys. 120, 9911 (2004).

${ }^{14}$ M. Amsler and S. Goedecker, J. Chem. Phys. 133, 224104 (2010).

${ }^{15}$ K. Bao, S. Goedecker, K. Koga, F. Lançon, and A. Neelov, Phys. Rev. B 79, 041405 (2009).

${ }^{16}$ M. Amsler, J. A. Flores-Livas, L. Lehtovaara, F. Balima, S. A. Ghasemi, D. Machon, S. Pailhès, A. Willand, D. Caliste, S. Botti, A. S. Miguel, S. Goedecker, and M. A. L. Marques, Phys. Rev. Lett. 108, 065501 (2012).

${ }^{17}$ S. Botti, J. A. Flores-Livas, M. Amsler, S. Goedecker, and M. A. L. Marques, Phys. Rev. B 86, 121204 (2012).

${ }^{18}$ J. A. Flores-Livas, M. Amsler, T. J. Lenosky, L. Lehtovaara, S. Botti, M. A. L. Marques, and S. Goedecker, Phys. Rev. Lett. 108, 117004 (2012).

${ }^{19}$ M. Amsler, J. A. Flores-Livas, T. D. Huan, S. Botti, M. A. L. Marques, and S. Goedecker, Phys. Rev. Lett. 108, 205505 (2012).

${ }^{20}$ T. F. T. Cerqueira, R. Sarmiento-Pérez, F. Trani, M. Amsler, S. Goedecker, M. A. L. Marques, and S. Botti, MRS Commun. 3, 157 (2013).

${ }^{21}$ R. Sarmiento-Pérez, T. F. T. Cerqueira, I. Valencia-Jaime, M. Amsler, S. Goedecker, S. Botti, M. A. L. Marques, and A. H. Romero, New J. Phys. 15, 115007 (2013).

${ }^{22}$ G. Kresse and J. Furthmüller, Comput. Mater. Sci. 6, 15 (1996).

${ }^{23}$ J. P. Perdew, K. Burke, and M. Ernzerhof, Phys. Rev. Lett. 77, 3865 (1996).

${ }^{24}$ S. Curtarolo, W. Setyawan, S. Wang, J. Xue, K. Yang, R. H. Taylor, L. J. Nelson, G. L. Hart, S. Sanvito, M. Buongiorno-Nardelli, N. Mingo, and O. Levy, Comput. Mater. Sci. 58, 227 (2012).

${ }^{25}$ J. E. Saal, S. Kirklin, M. Aykol, B. Meredig, and C. Wolverton, JOM 65, 1501 (2013).

${ }^{26}$ J. P. Perdew, A. Ruzsinszky, G. I. Csonka, O. A. Vydrov, G. E. Scuseria, L. A. Constantin, X. Zhou, and K. Burke, Phys. Rev. Lett. 100, 136406 (2008).

${ }^{27}$ J. Heyd, G. E. Scuseria, and M. Ernzerhof, J. Chem. Phys. 118, 8207 (2003).

${ }^{28}$ J. Heyd, G. E. Scuseria, and M. Ernzerhof, J. Chem. Phys. 124, 219906 (2006).

${ }^{29}$ H. Okamoto, J. Phase Equilib. Diffus. 33, 500 (2012).

${ }^{30} \mathrm{~T}$. Uesugi, Y. Takigawa, and K. Higashi, Mater. Trans. 46, 1117 (2005).

${ }^{31}$ See supplementary material at http://dx.doi.org/10.1063/1.4905141 for phonon band structures of all stoichiometries..

${ }^{32}$ R. Hill, Proc. Phys. Soc., London, Sect. A 65, 349 (1952).

${ }^{33}$ J. Thomas, Phys. Rev. 175, 955 (1968).

${ }^{34}$ J. Trivisonno and C. Smith, Acta Metall. 9, 1064 (1961).

${ }^{35}$ P. A. Korzhavyi, A. V. Ruban, S. I. Simak, and Y. K. Vekilov, Phys. Rev. B 49, 14229 (1994).

${ }^{36}$ M. H. F. Sluiter, Y. Watanabe, D. de Fontaine, and Y. Kawazoe, Phys. Rev. B 53, 6137 (1996).

${ }^{37}$ Y. Qi, L. Hector, C. James, and K. Kim, J. Electrochem. Soc. 161, F3010 (2014). 